\title{
Amor, posmodernidad y perspectiva de género: entre el amor romántico y el amor líquido
}

\author{
Alejandro Sánchez-Sicilia ${ }^{1}$, Jenny Cubells Serra ${ }^{2}$
}

Recibido: Noviembre 2017 / Evaluado: Marzo 2018 / Aceptado: Abril 2018

Resumen. Bauman acuñó el término modernidad líquida para conceptualizar y visibilizar la nueva manera de relacionarnos y vincularnos interpersonalmente. En el trabajo que aquí presentamos hemos querido analizar esta transición, mediante análisis del discurso, en concreto de repertorios interpretativos del programa de adolescentes para la búsqueda del amor Chicos y Chicas de YouTube, identificando las características que conforman el repertorio del amor líquido tanto en sus elementos teóricos como retóricos. El análisis que hemos llevado a cabo nos ha permitido identificar a su vez el conflicto, convivencia, complementación y fusión entre el amor romántico y el líquido, produciéndose así un fenómeno al que hemos denominado Consumo Romántico. Desde el análisis de género, tanto las prácticas románticas como las líquidas suponen una subordinación y objetivización de lo femenino frente a lo masculino. En ambas prácticas las mujeres son desprovistas de su capacidad de agencia, y se las presenta como entes a la espera de ser conquistadas en el amor romántico, y consumidas en el amor líquido. Palabras clave: Discurso; Bauman; Estudios de Género; Adolescentes; Consumo

\section{[en] love, postmodernity and gender perspective: between romantic love and liquid love}

\begin{abstract}
Bauman coin the term liquid modernity to conceptualize and visualize the new form that we use to implicate and link us interpersonally. In this article we have wanted to analyze this transition, by means of discourse analysis, in concrete of the interpretative repertories of the searching love program Boys and Girls issued on the YouTube platform, identifying the characteristics that make up the liquid love repertoire, in both cases, theoretical and rhetorical elements. The analysis we have carried out, has allowed to identify the conflict, coexistence, complementation and fusion between the romantic and liquid love, creating a phenomenon that we have named "Romantic Consumption". Likewise, from gender analysis, both romantic and liquid practices involve a subordination and objectification of the feminine before the masculine. In both practices, women are unprovided of their agency capacity, and they are presented as objects waiting to be conquered in romantic love, and consumed in liquid love.

Keywords: Discourse; Bauman; Gender Studies; Teenagers; Consumption
\end{abstract}

Sumario: 1. Introducción. 2. Metodología. 3. Resultados y discusión. 3.1. Emocionalidad/Racionalidad. 3.2. Compromiso/Libertad. 3.3. Sacrificio/Hedonismo. 3.4. Profundidad/Superficialidad. 3.5 Realidad/Virtualidad. 4. Conclusiones. 5. Referencias.

\footnotetext{
1 Universidad Autónoma de Barcelona sicilia_a@outlook.es

2 Universidad Autónoma de Barcelona jenny.cubells@uab.cat
} 
Cómo citar: Sánchez-Sicilia, A.; Cubells Serra, J. (2018). Amor, posmodernidad y perspectiva de género: entre el amor romántico y el amor líquido, Investigaciones feministas 9.1, 151-171.

\section{Introducción}

La obra de Bauman en lo que a la última década se refiere, se ha caracterizado y se caracteriza por tomar como referencia y foco de análisis la modernidad líquida, término acuñado por él mismo, el cual surge del movimiento posmodernista, socavando el proyecto modernista y subvirtiendo, simultáneamente, las concepciones románticas del yo (Gergen, 2006), donde el consumo se torna protagonista y emerge de manera interseccional siendo aplicable a múltiples contextos más allá de las relaciones interpersonales amorosas en las cuales se centra este trabajo. Entre sus obras destacamos aquella que, por su profundidad y concreción de análisis sobre los vínculos interpersonales ha sido nuestra guía de referencia y nos ha permitido llevar a cabo este trabajo, Amor líquido (Bauman, 2003). En la modernidad líquida, como recoge Eva Illouz (2009) la forma de relacionarnos se ha impregnado del sistema capitalista. En la sociedad de consumidores, las relaciones interpersonales se constituyen a imagen y semejanza de las relaciones que se establecen entre consumidores y objetos de consumo; somos simultáneamente los promotores del producto y el producto que promueven; la fuerza de trabajo debe estar siempre en óptimas condiciones para atraer la mirada de potenciales compradores (Bauman, 2007). En la sociedad de consumo impregnada por el sistema capitalista, las personas nos convertimos en agentes y entes de consumo. Bauman (2007) hace referencia al modelo neoliberal del cual se impregna la modernidad líquida, a través del cual sostiene que el consumismo es el acuerdo social que resulta de la reconversión de los deseos, ganas y/o anhelos humanos en la principal fuerza de impulso y de operaciones de la sociedad. Deseo, ganas, anhelos, son características de la modernidad líquida, basadas en la búsqueda de la satisfacción inmediata de dichas necesidades.

Nuestra investigación parte de la perspectiva socio-construccionista de Kenneth y Mary Gergen (2007, 2011), el conocimiento situado, postura epistemológica crítica, que propone especificar y ser conscientes de la posición desde la que se investiga (Donna Haraway, 1988) y la perspectiva de género (Wollstonecraft, 1792; De Beauvoir, 1949/1969; Friedan, 1963; Lee Bartky, 1990; Jónnasdóttir, 1996; Hooks, 2000; Olsen, 2000; Smart, 2000; Bourdieu, 2001 y Coral-Díaz, 2010; Medina Doménech, 2012; Cubells y Calsamiglia, 2016; Sánchez-Sicilia y Cubells, 2017; ). Desde la perspectiva construccionista, el discurso constituye la realidad, no la describe -es lo que se llama epistemología no-representacionista- (Harré, 1986; Harré \& Parrott, 1996). Michel Foucault (1992), nos hace conscientes de cómo estos discursos coaccionan, limitando los poderes, dominando las apariciones aleatorias, y seleccionando a los sujetos que pueden hablar.

Nos interesa conocer cómo se construye el sentimiento amoroso en la adolescencia, pues es en esta etapa donde tienen lugar las primeras relaciones sexo afectivas y emergen las prácticas, mitos y/o repertorios amorosos que posibilitan la aparición y mantenimiento de la violencia de género en las relaciones. Es en estos grupos de iguales donde se establecen las primeras pautas y relaciones amorosas entre sus 
miembros. Estas pautas están mediatizadas por unos discursos que orientan a los adolescentes sobre cómo desenvolverse en las primeras relaciones de pareja que constituyen. Chicos y Chicas es un programa de búsqueda del amor para adolescentes, orientado bajo el repertorio del amor romántico (Cubells y Calsamiglia, 2016; Sánchez-Sicilia y Cubells, 2017), donde acuden jóvenes con la intención de conocerse y establecer en fin último una relación de pareja. Se trata de un material transmitido por la plataforma YouTube en España, con una amplia acogida por parte del público adolescente, con un total de un millón de visualizaciones de media en la temporada que analizamos. Es por ello que nos decantamos por analizar este programa, por su amplia acogida y por su capacidad para hacer, deshacer, construir y transmitir discursos a los cientos de miles de adolescentes que siguen el programa.

Los estudios del amor con perspectiva de género recogen que a la mujer todo la incita a dejarse sitiar y dominar por existencias extrañas, y singularmente en el amor, se niega en vez de afirmarse (De Beauvoir ,1949/1969, 407). Betty Friedan (1963) en la mística de la feminidad presenta como este malestar que subordina a las mujeres, se edulcora con las panaceas tradicionales del amor es la respuesta. Anna Jónnasdóttir (1996) sostiene que la organización social del amor es la base del patriarcado occidental contemporáneo, y que la mujer para realizarse como persona necesita amar y ser amada para habilitarse socio-existencialmente. Bell Hooks (2000) nos presenta el desamor como una preocupación colectiva para las mujeres, como muchas mujeres creen que nunca conocerán un amor pleno, están dispuestas a conformarse con estrategias que alivien el dolor y acrecienten la paz, el placer y la alegría en sus relaciones existentes, en especial las sentimentales. Rosa María Medina Doménech (2012) sostiene que el amor, al ser tema de trabajo de hombres, se convierte en política emocional para el sometimiento de las mujeres. En ese espacio, las mujeres quedaban presas de las normativas del amor. Ana de Miguel (2015) expresa que las mujeres no solo invierten demasiadas energías de sus vidas en el amor, sino que, sobre todo, acaban subordinando sus proyectos de vida a los de sus parejas. Las mujeres están tan dispuestas a todo por triunfar en el amor-o por no reconocer su fracaso-, que son incluso capaces de soportar situaciones diversas de violencia.

En nuestra investigación, analizaremos los valores patriarcales y prácticas discriminatorias por género que fomentan las formas románticas y líquidas. Como recogen Jenny Cubells y Andrea Calsamiglia (2016) y Alejandro Sánchez-Sicilia y Jenny Cubells (2017) en sus estudios, el repertorio del amor romántico orienta las formas adecuadas (normativas) de sentir, pensar y actuar, favoreciendo las relaciones heterosexuales, románticas, monógamas y duraderas. Así, genera subjetividades convenientes para la reproducción y mantenimiento del heteropatriarcado. Mari Luz Esteban y Ana Távora (2008) sostienen que el amor romántico es parte intrínseca de la subordinación de las mujeres. De esta manera ponen de manifiesto la construcción de la desigualdad entre hombres y mujeres en el proceso de socialización en el amor en el que se atribuye roles y expectativas distintas en función del género (Esteban y Távora, 2008; Gil y Lloret, 2007; Pujal, 2007). En el amor líquido (Bauman, 2003), la premisa parte de estrechar lazos relacionales, pero manteniéndolos al mismo tiempo débiles para poder desanudarlos. Es decir, se establecen vínculos formados por personas desesperadas por relacionarse, pero que desconfían continuamente del estar relacionadas, sobre todo cuando se habla de estarlo para siempre o eternamente, pues temen la carga que puede suponer este estado con el cual no se sienten capaces ni deseosos de soportar, además que este estado puede limitar la libertad que nece- 
sitan para relacionarse con otros. Un aspecto fundamental a tener en cuenta es que Bauman obvia el género en su teoría del amor líquido, y es por ello que nos hemos sentido interpelados a incorporarlo en este trabajo. Autoras como Ana de Miguel (2015) hacen hincapié en que la mayoría de los autores que han estudiado el establecimiento de los vínculos en la sociedad posmoderna, no tienen una perspectiva de género e ignoran la visión específica de las mujeres, quienes experimentan una continua decepción en sus relaciones y falta de reciprocidad entre lo que aportan y lo que reciben. Alejandra Kollontai (1976) un siglo atrás, ya ponía en duda que un cambio en los lazos formales o externos que unen a la pareja, supusiera un cambio en la relación desigual entre hombres y mujeres a nivel afectivo sexual. Consideraba que una unión libre debía basarse en el mutuo respeto de la individualidad y de la libertad del otro. Implica rechazar la subordinación de las mujeres dentro de la pareja y de la hipocresía de la doble moral sexual. Asimismo, Marcela Lagarde (2001) sostiene que la revolución no está, de forma intrínseca, en la mera forma de las relaciones, sino en que las mujeres lleguen a ser sujetos de pacto y negociación en las relaciones amorosas.

\section{Metodología}

Nuestro estudio se enmarca en la perspectiva socio construccionista, es por ello, que la metodología a utilizar es de corte cualitativo, a través de la interpretación, pretendemos comprender la naturaleza de los procesos sociales más que explicarla o predecirla (Iñiguez, 2008). Desde la perspectiva socio construccionista, al comunicarnos unos con otros construimos el mundo en el que vivimos, nuestras acciones no están condicionadas por nada tradicionalmente aceptado como verdadero, lógico o correcto (Gergen y Gergen, 2011). El construccionismo entiende el conocimiento como el producto de comunidades determinadas, guiadas por supuestos, creencias y valores concretos. No existe la Verdad (con mayúsculas) para todos sino la verdad (con minúsculas) de cada comunidad (Gergen, 2007).

No podemos separar el análisis de las prácticas amorosas del análisis de género, $\mathrm{y}$ es por ello que hemos incorporado y adoptado la perspectiva del sistema dual masculino/femenino (Bartky, 1990; Olsen, 2000; Smart, 2000; Bourdieu, 2001 у Coral-Díaz, 2010). En cada par del dualismo, el término femenino es identificado como negativo, corrupto o inferior, mientras que el término masculino, es privilegiado como superior (Olsen, 2000). Frances Olsen (2000) sostiene que los hombres se han identificado a sí mismos con un lado del dualismo: con lo racional, lo activo, el pensamiento, la razón, la cultura, el poder, lo objetivo, lo abstracto, lo universal. Por el contrario, las mujeres resultaron proyectadas hacia el otro lado e identificadas con lo irracional, lo pasivo, el sentimiento, la emoción, la naturaleza, la sensibilidad, lo subjetivo, lo concreto, lo particular. La experiencia femenina ha sido jerarquizada y dominada en este binomio polarizado sujeto masculino neutro/feminidad. En el entramado occidental del dualismo, las mujeres se convierten en lo que los hombres no son (Coral-Díaz, 2010). Sandra Lee Bartky (1990) emplea el término objetivación sexual al referirse a la definición de una persona por sus partes o funciones sexuales, al separarlas del resto de su personalidad, y de esta forma, aislarla del todo y reducirla a instrumentos que logran representar al sujeto dominado. Continuando en esta 
misma línea de la corporeidad como sustentadora de este dualismo diferenciador y jerarquizado, Carol Smart (2000) sostiene que la mujer además de constituir uno de los lados de la diferenciación binaria anterior, representa el dualismo, en el sentido de que en el discurso jurídico, la prostituta es construida como la mala mujer, pero al mismo tiempo se erige en el epítome de la Mujer en contraposición al Hombre, porque es lo que cualquier mujer podría ser, y porque representan una tortuosidad y un libertinaje surgidos de su forma corporal (que se supone naturalmente dada), mientras que el hombre permanece inofensivo. Bourdieu (2001) continúa en esta línea y sostiene que las construcciones sobre masculinidad y feminidad se escriben sobre el cuerpo, que de alguna manera está relacionado con el uso de la fuerza, un ejemplo de ello sería hablar de la fuerza superior de la masculinidad, frente a la fragilidad física y emocional femenina.

El análisis de los datos se fundamenta en la teoría recogida por Bauman en su trabajo Amor líquido (Bauman, 2003). En primer lugar, a partir de esta teoría, hemos realizado un análisis de contenido, definido este como una técnica de investigación destinada a formular, a partir de ciertos datos, inferencias reproducibles y válidas que puedan aplicarse a su contexto (Krippendorff, 1980 ), y en concreto un análisis de contenido categorial temático, que como recoge Félix Vázquez (1996), trata de ir descomponiendo el texto en unidades, para proceder posteriormente, a su agrupamiento en categorías, considerando las similitudes y semejanzas que existan entre estas en función de criterios previamente establecidos según los objetivos de investigación y/o de análisis (criterio de analogía). El análisis de la teoría Amor líquido de Bauman (2003), nos ha permitido descomponer su trabajo en 5 ejes y 10 categorías. Cada eje está compuesto por dos categorías opuestas, referentes al amor romántico y al amor líquido. El proceso que seguimos consistió en la lectura analítica de su trabajo, donde íbamos señalando aquellas citas que hacían referencia a esta convivencia/transición entre las formas románticas y las formas líquidas en las relaciones. A medida que íbamos señalando estas citas, iban emergiendo de ellas categorías, que a medida que iba avanzando el análisis se iban repitiendo, aunque con citas diferentes, hasta constituirse en lo que en un primer momento consideramos como 9 ejes y 18 categorías diferenciadas. Tras su discusión, fueron incluidas y simplificadas en los 5 ejes y 10 categorías comentadas anteriormente, y desarrolladas en el apartado de resultados y discusión.

En segundo lugar, una vez descompuesta la teoría completa en ejes y categorías, hemos procedido a analizar la transcripción del programa chicos y chicas.

Chicos y chicas es un programa de búsqueda del amor para adolescentes, emitido íntegramente por la plataforma YouTube en España, en el cual participan adolescentes de edades comprendidas entre los 14 y los 19 años, y no adolescentes, pero que contribuyen también a reproducir y construir discursos en torno a la temática abordada en el programa. El programa lo componen en orden jerárquico (entendiendo este como quien o quienes otorgan el derecho a hacer y/o deshacer en él) de arriba a abajo:

- Propietarios de los derechos, director y equipo técnico.

- Presentador que dinamiza y marca los tiempos de intervención.

- Consejeros del amor que realizan preguntas, intervienen y en algunas ocasiones marcan los tiempos de intervención.

- Tronistas, adolescentes de ambos sexos que reciben pretendientes/as que vienen a conquistarlos. 
- El público del plató, compuesto en su mayoría por adolescentes de sexo masculino, que interviene como unidad o a través de miembros del mismo, manifestando su opinión con lo que en el programa sucede.

- Pretendientes/as, adolescentes de ambos sexos que van a conquistar a los y las tronistas.

- Por último, podemos considerar parte activa del programa a los espectadores, los cuales, con sus críticas y comentarios van modificando el contenido del programa.

El programa se encuentra totalmente accesible para cualquier persona que desease visionarlo. Asimismo, el artículo 32 del Real Decreto Legislativo 1/1996, de 12 de abril, considera en lo que a nuestro caso se refiere:

Lícita la inclusión en una obra propia de fragmentos de otras ajenas de naturaleza escrita, sonora o audiovisual (...) siempre que se trate de obras ya divulgadas y su inclusión se realice a título de cita o para su análisis, comentario o juicio crítico. Tal utilización solo podrá realizarse con fines docentes o de investigación (...).

Estudiar un entorno virtual o digital no está exento de polémica, pues se ha mantenido la idea de que aquello que es virtual, marca una polaridad frente a real, dando a entender que las relaciones que se estudian son menos reales que las que mantenemos físicamente (Estalella y Ardévol, 2007) En nuestro caso, los aspectos fuera de cámaras no cobran demasiada importancia pues son precisamente los aspectos que son reproducidos, los que nos interesan por su alcance y su capacidad discursiva.

La trascripción ha sido llevada a cabo mediante el visionado completo de la primera temporada del programa, disponible en la plataforma YouTube. Hemos transcrito 9 capítulos compuestos por un total de 185 minutos de material analizable. La transcripción ha sido llevada a cabo siguiendo la adaptación del código de Jefferson para las ciencias sociales (Follari, 2015). Nuestra labor analítica, se ha basado en recoger aquellas referencias textuales que pertenecen a una categoría concreta y por ende a un eje y repertorio específico. Para ello, hemos realizado varias lecturas del material, y hemos ido señalando y recogiendo mediante un sistema numérico, las citas textuales en función de la categoría y eje al que se adscriben. Una vez seleccionadas las referencias, hemos llevado a cabo un análisis del discurso en concreto de repertorios interpretativos. En palabras de Iñiguez y Antaki (1994), el análisis de discurso refiere a:

El conjunto de prácticas lingüísticas que mantienen y promueven ciertas relaciones sociales. El análisis consiste en estudiar cómo estas prácticas actúan en el presente manteniendo y promoviendo estas relaciones: es sacar a la luz el poder del lenguaje como una práctica constituyente y regulativa (Iñiguez y Antaki, 1994).

Con la intención de explicar aquellos mecanismos lingüísticos a los que recurrimos con el fin de construir nuestras versiones sobre el mundo, llevaremos a cabo un análisis de repertorios interpretativos (Potter y Wetherell, 1987). De esta forma, los repertorios interpretativos se dibujan como estrategias encaminadas a construir, con una finalidad concreta, una representación determinada de la realidad. Para comprender el fenómeno que aquí analizamos, estudiamos la función a través del estudio 
de la variabilidad en la construcción de los discursos (Wetherell y Potter, 1996). La variabilidad es un índice de la función, como un índice de las distintas maneras en que se puede fabricar una explicación. La construcción nos guía hasta el lugar en el que el discurso se fabrica a partir de recursos lingüísticos preexistentes con características propias (Wetherell y Potter, 1996). Las funciones que encontramos en el análisis corresponden a formas de justificación, rechazo, apoyo ... que emergen gracias a la interrelación que mantienen con las variaciones del lenguaje, a su poder de construir y reproducir un discurso y a los repertorios interpretativos que destacan.

Siguiendo la metodología propuesta por Wetherell y Potter (1996), es preciso llevar a cabo una serie de codificaciones preliminares para poder trabajar con un grupo de datos manejable. El estudio de las funciones, variaciones y construcciones se llevará a cabo gracias a las técnicas argumentativas y retóricas que nos ofrece el tratado de la argumentación de Perelman \& Olbrechts-Tyteca (1989), así como al estudio de las metáforas de Lizcano (1999; 2006). A lo largo del análisis, a medida que vayan surgiendo estas técnicas serán resaltadas en el texto, definida su función y asociada al extracto de la referencia en la cual las hemos observado. Este análisis ha permitido identificar patrones recurrentes cuanto a la temática y a la forma del discurso que caracterizan a los repertorios.

El orden que seguirá el análisis será el siguiente: presentaremos cada eje; las categorías opuestas; la teoría a las que hacen referencia; las citas textuales que a modo de ejemplo sustentan y constituyen estas categorías y por ende estos ejes y repertorios; y por último el análisis de repertorios interpretativos de dichas citas. Asimismo, de manera transversal, iremos analizando desde la perspectiva de género, como se presenta y es presentada la mujer en estas interacciones.

Somos conscientes que muchos de los ejemplos que presentamos para cada categoría, podrían formar parte también de otras categorías del repertorio al que se adscriben, lo cual cobra sentido del hecho de que pertenecen en su fin último al mismo repertorio, y son categorías que están relacionadas, y todas juntas lo conforman. Aun así, en aras de presentar un análisis claro y que no suscite confusión al lector, hemos querido presentarlas como ejemplos de una categoría concreta, aquella de la cual consideramos es más representativa y evitar la repetición de una misma referencia varias veces. Consideramos que la metodología que hemos seguido mostrará de forma clara y precisa aquello que pretendemos transmitir.

\section{Resultados y discusión}

A lo largo del análisis hemos identificado dos repertorios, por un lado, el repertorio del amor romántico, recogido ya por Jenny Cubells y Andrea Calsamiglia (2016) y Alejandro Sánchez-Sicilia y Jenny Cubells (2017) en sus trabajos. El repertorio del amor romántico mediante el uso de metáforas muertas (Lizcano, 1999), mecanismos de exteriorización (Woolgar, 1988) y estrategias retóricas que amplifican el sentimiento (Anita Pomerantz, 1986) construye el amor como un fenómeno externo que escapa del control y la voluntad de las personas y que se presenta como un elemento central en la vida de las personas. Así mismo, el repertorio del amor romántico establece las formas correctas de sentir, pensar y actuar, favoreciendo las relaciones heterosexuales, románticas, monógamas y duraderas, y generando las condiciones de 
posibilidad para la violencia machista en el seno de la pareja. Por otro lado, emerge de este análisis el repertorio del amor líquido, donde destacan las prácticas líquidas, relaciones libertarias, superficiales y hedónicas, abordadas de forma racional, siendo prácticas comunes en las relaciones virtuales. Estas características se hacen presentes mediante el uso generalizado de figuras y argumentos consideradas racionales o en la búsqueda de objetividad. Entre ellas destacamos los lugares de lo existente, los lugares de cantidad, los razonamientos basados en las probabilidades, los argumentos pragmáticos y los argumentos a fortiori. Aunque en menor frecuencia que las anteriores, destacamos también el uso de argumentos de lo irreparable, prolepsis, polarización de virtudes y vicios, enlaces de coexistencia de acto-persona, y enlaces de sucesión del fin y lo medios.

A continuación, presentaremos cada eje, con sus respectivas dos categorías enfrentadas, una característica del amor romántico y otra del amor líquido. Acto seguido presentaremos el fragmento de la teoría de Bauman (2003) a partir de la cual hemos desarrollado estas categorías. A continuación, las intervenciones de los adolescentes y miembros componentes del programa, las cuales serán analizadas a través de las figuras retóricas y de argumentación que nos aportan Perelman \& Olbrechts-Tyteca (1989) y el estudio metafórico de Lizcano (1999; 2006) atendiendo a las variaciones, construcciones y funciones que derivan del análisis de repertorios interpretativos con perspectiva de género, como comentábamos con anterioridad.

\subsection{Emocionalidad/Racionalidad}

En una relación de bolsillo (...) debe embarcarse en la relación con total conciencia y claridad. Recuerde, nada de amor a primera vista. Nada de enamorarse... Nada de esas súbitas mareas de emoción que lo dejan sin aliento: nada de esas emociones que llamamos amor ni de esas a las que sobriamente denominamos deseo. Usted no debe permitir que nadie le arrebate la calculadora de la mano (Bauman, 2003, 38) (...) Las consideraciones técnicas no se llevan bien con las emociones (Bauman, 2003, 69).

Referencias a la contraposición entre amor romántico (emoción) y amor líquido (racional) se hacen manifiestas a lo largo del análisis. En primer lugar, presentamos aquellas citas que denotan emocionalidad/irracionalidad:

(...) viene::: con ganas de guerra (1) viene e::: dispuesta a darlo todo, y sobre todo a comerse a sus pretendientes (...) (haciendo referencia a Tronista Laura) (presentador)

Pretendiente Mateu tronista Paula.: Y voy a todas en una relación (...)

Tronista Paula.: Tenía algo que me gustaba (1) tenía y lo, lo sigue teniendo (...)

La idea del amor como algo irracional, emocional, se hace manifiesta mediante el empleo de metáforas muertas, pues ya no se perciben como metáfora, sino como un concepto bien definido, como la realidad misma. Las metáforas muertas lejos de desactivar la potencia metafórica, la refuerza, pues al mantenerla inconsciente, hace imposible el control sobre la ficción que la instituye, darlo todo, ir a todas en una relación, o comerse a sus pretendientes, entendidas estas a su vez con sentido de 
hipérbaton, amplificando y haciendo énfasis en la idea que pretende transmitir; o como ese algo, recibe la valoración de lo concreto y el valor dado a lo único, típico de los lugares de cualidad, ese algo, que la tronista no es capaz de explicar, de razonar, desvela el carácter único de algo, lo único es original, se distingue, destaca y agrada, siendo valorizado por el hecho mismo. Estas referencias al amor como acto irracional o inexplicable se contradicen con la idea racional que a continuación, se presenta:

Pretendiente Sergi tronista Paula.: Ante todo quiero dejar claro que me quiero ir (...) somos cuatro, hay poquísimas (x) prob- posib- probabilidades (...)

个Tú tienes que tener cabeza y irte $\uparrow$ (Público Luis)

Yo de vosotros me iría ya (...) ¿y el orgullo chavales?, iros de aquí (...) (Público Cristian)

Las referencias anteriores bien podrían emplearse como idóneos ejemplos de esta forma que Bauman $(2003,38)$ tenía para referirse a las decisiones tomadas bajo la vía de lo racional Usted no debe permitir que nadie le arrebate la calculadora de la mano. Observamos varias premisas y figuras en estas referencias. En primer lugar, destacar el empleo de los lugares de lo existente en los tres ejemplos, al confirmarse la superioridad de lo que existe, de lo que es actual, de lo que es real, sobre lo posible, lo eventual o imposible. Esto lo apreciamos en cómo los pretendientes se apoyan en esta superioridad de lo actual o real, que sucede, para abandonar el programa. Un claro ejemplo sería la cita somos cuatro, hay poquísimas probabilidades, el cual no solo encaja en el caso anterior, sino que es remitida también a los lugares de canti$d a d$, donde se aprecia una clara preferencia dada a lo probable sobre lo improbable; asimismo, la aplicación de razonamientos basados en las probabilidades tendrá por resultado, el dar a los problemas un carácter empírico, por tanto justifica y asegura esta acción futura, en este caso abandonar el programa a través de una prolepsis, anticipándose a las posibles objeciones que puedan realizarle a su argumento. El pretendiente emplea un argumento pragmático, al proponer el éxito de su toma de decisiones como criterio de objetividad, de validez, el cual contrasta totalmente con la expresión vengo a darlo todo de la categoría emocionalidad/irracionalidad en el amor. Apreciamos en esta categoría esta vez, un ejemplo de metáfora muerta en la expresión tener cabeza, la cual refiere en este caso al uso racional en la toma de decisiones en el amor. Para finalizar, en la última referencia, el uso de la expresión interrogativa ¿y el orgullo chavales?, cuando ya previamente había mostrado su parecer respecto a continuar o no en el programa, muestra un ejemplo de epanortosis, cuyo uso amplifica el sentido de una expresión anteriormente expuesta.

Por último, nos gustaría presentar dos situaciones, donde en cada una de ellas, se presenta esta contraposición de categorías:

Público Cristian.: La Paula (...) tenía una $P$, tatuada en el pecho.

Presentador.: ¿En el pecho tienes una P tatuada?

Tronista Paula.: No, me lo hice con lo de los ojos

Público Cristian.: (...) era una P de Pau, era una P de Pau

Tronista Carles.: (...) fíjate bien en ella

Tronista Alex.: (:)Si, lleva un colgante con mi nombre:; 
Consejera.: ¿Ah sí?

Tronista Alex.: :-) Y si, es muy bonito $:$;

Consejero.: ¿Y por qué llevas un colgante con su nombre?

Pretendienta Laia tronista Alex.: Lo veo cada dos semanas y (2) quiero algo que me recuerde a él cada día

Consejera.: Muy bien (2) encima, encima romántica

En las dos situaciones presentadas anteriormente, ambas embargadas por la emoción y la irracionalidad propias del amor romántico se tatúan en el pecho la inicial del chico que le gusta, o portan un colgante con el nombre de su tronista. Si no profundizásemos más en estas situaciones, aceptaríamos que ambos actos pertenecen claramente al repertorio del amor romántico, pero al ahondar en ellos, emergen características a su vez propias del repertorio líquido. Esto lo apreciamos en la superficialidad, fugacidad del efecto de estos actos en el tiempo, el enlace simbólico presentado entre el símbolo de la inicial plasmada con un lápiz de ojos, o como el colgante, y el ser amado como simbolizado, pueden dejar de tener su significado, cuando decidan borrarse la inicial o quitarse el colgante. Por tanto, un acto que muestra a su vez características emocionales/irracionales y superficiales/racionales, nos lleva a considerar que se trata de una complementación entre el repertorio romántico y el líquido. En este caso al contrario de lo que diría Bauman, las consideraciones técnicas si se llevan bien con las emociones, se complementan. Asimismo, nos gustaría prestar especial atención a la intervención de la consejera del amor, Muy bien encima, encima romántica, la cual mediante un juicio de valor refuerza la máxima del amor romántico, la cual goza de valor y respaldo popular. Intervención avalada por ser un argumento de autoridad, de prestigio, y por tanto, con mayor poder para transmitir unos discursos, unas máximas concretas, además de utilizar la repetición para duplicar el efecto de presencia.

Desde un análisis de género, no nos sorprende que en su amplia mayoría las referencias al comportamiento irracional o emocional del amor romántico, que hemos observado anteriormente comerse a sus pretendientes, darlo todo, las estrategias de venta románticas (en este caso complementadas con características líquidas) estén asociadas a las mujeres y al polo femenino. Asimismo, todas las expresiones racionales asociadas al amor líquido pertenecen a hombres, tienes que tener cabeza, ¿y el orgullo chavales? Esto tiene su raíz en la concepción de los dualismos masculino/femenino (Olsen, 2000) donde se ha asociado culturalmente al hombre con lo racional, el pensamiento, lo objetivo y a la mujer con lo irracional, el sentimiento, la emoción.

\subsection{Compromiso/Libertad}

Bauman (2003) al inicio de su trabajo recoge la necesidad actual de las personas de estrechar lazos, pero manteniéndolos al mismo tiempo flojos para poder desanudarlos.

Desconfían todo el tiempo del estar relacionados y particularmente de estar relacionados para siempre, por no hablar de eternamente, porque temen que ese estado pueda convertirse en una carga y ocasionar tensiones que no se sientan capaces ni deseosos de soportar, y que pueden limitar severamente la libertad que necesitan- sí, usted lo ha adivinado- para relacionarse... (Bauman, 2003, 8). (...) 
El compromiso a largo plazo es una trampa que el empeño de relacionarse debe evitar a toda costa (Bauman, 2003, 10).

A continuación, presentamos referencias que apoyan el compromiso del amor romántico, y por tanto la exclusividad a la pareja:

Pretendiente Mateu tronista Paula.: Y voy a todas en una relación (...) cuando estoy en una relación voy con la mente pensando en ella (Incomprensible, 2)

Tronista Paula.: Eso me gusta eh

Presentador.: <Genial, o sea tu tiempo, o sea tú eres para ella, solo para ella, todo tuyo para ella>

Pretendiente Mateu tronista Paula.: Si

El chico ideal ya no lo necesito (1) solo necesito que::: me traten bien, que sepan cuidarme y que estén solo por mi (Tronista Laura)

Tronista Laura.: Quiero tener los justos, no quiero tener muchos, agobiarme y que luego salga mal

En primer lugar, antes de comenzar con el análisis de las referencias, nos gustaría destacar que el compromiso, la lealtad o fidelidad, son nociones que pertenecen a los valores concretos, de ahí que su valor sea puesto en alza, al entenderse estos como actos con valor de unicidad. En la primera cita expuesta, se emplean metáforas muertas que denotan la irracionalidad propia del amor romántico vista en la categoría anterior, pero esta vez añadida a la noción de compromiso, de exclusividad voy con la mente pensando en ella. El compromiso fuera del repertorio romántico no es sinónimo de exclusividad, pero la exclusividad (propia del repertorio romántico) requiere de compromiso. Por tanto, en este eje en la categoría de compromiso expuesta aquí entendida como una característica romántica, emplearemos la noción de exclusividad complementaria al compromiso. Ante la intervención del pretendiente, su tronista corrobora este compromiso de exclusividad expuesto, eso me gusta eh. Asimismo, el presentador, figura que goza de prestigio, y por ende de argumento de autoridad, corrobora la máxima del compromiso y exclusividad como valor a tener en cuenta, por el carácter de unicidad expuesto anteriormente. A modo de perifrasis, mediante el empleo de la epanortosis amplifica con las expresiones o sea tu tiempo, o sea tú eres para ella, solo para ella, todo tuyo para ella la idea anteriormente expuesta por el pretendiente, del mismo modo, el empleo de la repetición para ella, duplica el efecto de presencia al continuar haciendo hincapié en el compromiso, exclusividad. En la segunda referencia, observamos el uso de la epanortosis para amplificar la expresión de no necesitar el chico ideal, haciendo referencia a la necesidad única de cuidado y compromiso de exclusividad. Esta necesidad de cuidado es recogida por Bourdieu (2001) cuando sostiene que las construcciones sobre masculinidad y feminidad se escriben sobre el cuerpo, que de alguna manera está relacionado con la forma como se ha propuesto el uso de la fuerza, un ejemplo de ello sería hablar de la fuerza superior de la masculinidad, frente a la fragilidad física y emocional femenina. En la última referencia, el argumento pragmático de tener los justos propone el éxito de su toma de decisiones como criterio de objetividad, de validez, en este caso la racionalidad característica del repertorio líquido es traída a colación, para garantizar el compromiso de exclusividad característico del repertorio romántico. 
En contraposición a los ejemplos anteriores, encontramos referencias que reflejan la necesidad de mantenerse libres y por tanto el rechazo al compromiso:

Consejero.: ¿Esperas encontrar aquí el amor de tu vida?

Tronista Alex.: De mi vida no creo((ríe)) pero= (incomprensible, 2)

No, que yo::: venía con una idea, o sea que yo::: no he venido aquí a perder el tiempo, ni por fama ni nada, que yo me voy porque estoy conociendo a otra chica fuera (...) (Pretendiente Víctor tronista Laura)

Que no quiero seguirla conociendo y ya está (3) no quiero que esté aquí si se va liando con otros (tronista Alex).

Pretendienta Carla tronista Alex.: Si es verdad que se ha saltado las normas (...).

Entre las normas del programa, diseñado bajo el repertorio del amor romántico, destaca mantener un compromiso de exclusividad hacia tu tronista o hacia tus pretendientes, según sea el caso, y en las tres últimas referencias anteriores no parece que esto se esté produciendo, al menos se está cuestionando, algo que debería ser incuestionable. Metáforas muertas como perder el tiempo denotan la carga de mantener el compromiso de exclusividad a sus pretendientes o a su tronista. Los lugares de lo existente confirman la superioridad de lo que existe, de lo que es real, sobre lo posible o lo eventual, por lo tanto, en este caso el pretendiente bajo esta premisa ha decidido racionalmente apostar por el valor seguro de exclusividad. Es una manera de saltarse las normas del programa, pero no se cuestiona pues pasa de un intento de relación romántica exclusiva, a una relación romántica exclusiva. Dentro de la polarización de virtudes y vicios, la libertad líquida es entendida como vicio, desprestigio; mientras que el compromiso romántico como una virtud, prestigio. Por ende, no es de extrañar que se desprestigie tanto a las referencias a la libertad líquida y se valore tanto al compromiso romántico. El rechazo a las formas líquidas, que conflictúan con la máxima de compromiso y exclusividad, que aparecen en el programa, se va liando con otros, se ha saltado las normas (normas de compromiso de exclusividad), a modo de argumento a fortiori, si ha mostrado características del repertorio líquido (desprestigiado), no dudará en volverlas a mostrar contrastan con la gran cantidad de ejemplos que hacen referencia a la aparición constante de las formas líquidas. Haciendo referencia a esto, las dos pretendientas que acudieron a conocer a tronista Carles en el primer programa, no aparecieron en el segundo. Y de las tres nuevas que entraron al segundo programa, solo una apareció en el tercero. Así mismo, a lo largo del programa, los adolescentes, van y vienen con completa libertad, y la exclusividad, pilar de la monogamia brilla por su generalizada ausencia. Observamos algunas referencias que critican abiertamente la libertad líquida con la que convive el compromiso romántico:

(...) sobretodo fidelidad, que no, (x) od- o sea soy muy celoso (...) porque últimamente tal como está todo, tanto ellas como nosotros ((resopla)) no te puedes fiar nada de nada de nada. (tronista Alex)

Con esta crítica, hace referencia a la incursión del repertorio líquido en la sociedad actual, por tanto, reconoce una esencia de comportamiento líquido, y muestra rechazo a aquellas personas que actúen en relación a dicha esencia. 


\subsection{Sacrificio/Hedonismo}

En esta época de transición hacia el amor líquido, las personas abogan por relaciones satisfactorias, pero procurando evitar el sufrimiento:

Los seres humanos esperan (...) degustar las dulces delicias de las relaciones evitando los bocados más amargos y menos tiernos; como lograr que la relación les confiera poder sin que la dependencia los debilite, que los habilite sin condicionarlos, que los haga sentir plenos sin sobrecargarlos... (Bauman, 2003, 9) (...) Los habitantes de nuestro moderno mundo líquido (...), ¿Qué clase de consejo están buscando realmente? ¿Cómo anudar la relación o cómo por si acaso- deshacerla sin perjuicio y sin cargos de conciencia? (Bauman, 2003, 12).

Observamos referencias al sufrimiento/sacrificio propio del amor romántico, por parte de nuestros adolescentes:

$\uparrow$ Tú no puedes estar ahí de brazos cruzados, tú tienes que venir aquí a luchar, a conseguirla $\uparrow \cdot$, te gusta ella(2) pues a por ella, a pelear (consejero)

El hecho de que acudan a un programa donde tienen que cumplir unas normas de exclusividad hacia sus tronistas, y en el caso de ser tronista, hacia sus pretendientes, es una forma de sacrificio, sacrificas tus posibles vínculos sexo-afectivos con otras personas, para dedicarte totalmente a tu tronista o a tus pretendientes. En esta referencia, el consejero del amor hace uso del argumento del despilfarro cuando invita al pretendiente a continuar con la obra, puesto que ya se ha comenzado, aceptado sacrificios que serían inútiles en caso de renunciar, es preciso proseguir en la misma dirección. Observamos referencias a la categoría hedonismo, caracterizadas por la evitación del sacrificio/sufrimiento por parte de los participantes:

Pretendienta Carla tronista Alex.: (...) <yo puedo coger y me puedo ir $>$, no te voy a seguir conociendo sabiendo que has hecho eso, no puedo confiar en ti (...)

Pretendienta Carla tronista Alex.: (...) dejas entrar a todas las guarras $<$ de de de $>$ todos lados y encima $\uparrow$ ipretendes que yo me quede $\uparrow$ ?

Encontrar pareja en el programa, como fin previsto, requiere de una elección de mdios, aquellos que requieren menos sacrificio para la consecución de este fin goza de un valor inherente, de cierta superioridad. En este caso, se desecha la elección de aceptar la inclusión de características líquidas en las personas que participan en el programa, requiere de menos sacrificio rechazarlas por el mero hecho de no cumplir con las características románticas. La pretendienta en la primera referencia construye la imagen de su tronista en función de sus actos, en este caso, actos líquidos tras verterse sobre este, rumores de haber estado con otra chica fuera del programa, estos actos construyen su imagen de persona líquida. Ser incluido en el repertorio líquido una vez, supone ser víctima de argumentos a fortiori, quién ha mentido, no dudará en volver a mentir, en este caso, quién ha mostrado características líquidas, no dudará en volver a mostrarlas, y esto conlleva el empleo del argumento de lo irreparable, al concederle carácter de unicidad al acontecimiento calificado de irreparable, es valorizado por el hecho mismo, en nuestro caso, se observa como algo irreparable, 
haber coqueteado con actos líquidos. En el eje anterior, en la categoría de libertad observamos como las pretendientas no tienen problema en asistir o no al programa, y en lo que a esta categoría se refiere, anudando y deshaciendo la relación sin perjuicio y sin cargos de conciencia, es decir, de forma hedónica.

\subsection{Profundidad/Superficialidad}

Como recoge Bauman (2003), cuando la calidad, no nos da sostén, tendemos a buscar remedio en la cantidad. Con ello, quiere decirnos que en la modernidad líquida las relaciones se caracterizan por un frágil y superficial establecimiento de los vínculos personales, en detrimento de las relaciones románticas profundas, donde ahondamos en conocer y establecer un vínculo con el otro.

Se observa rechazo hacia las formas líquidas de superficialidad y apoyo hacia las formas románticas profundas:

Invitada Carolina.: ¿Tú quieres conocerme?

Tronista Carles.: :) Yo si $;$

Invitada Carolina.: ¿Pero como amiga?

Tronista Carles.: ¿Se empieza por algo no((ríe))?

Invitada Carolina.: (...) no te fijes tanto::: en el físico, :-ique es importante ¿no?:-; pero que las conozcas realmente como son

Presentador.: ¿Has tenido alguna relación?

Pretendiente Oriol tronista Paula.: Si, pero seria seria no, (...) o sea, todo el mundo busca solo que:.: follar y todo eso (1) ¿sabes?

Consejera.: ¿Diferente por qué? (...)

Pretendienta Laia tronista Alex.: Porque no me considero:.: como (2) como una guarra de las tantas que hay

Tronista Carles.: ((ríe)) (-)ha llamado a todas las niñas guarras:)

Consejera.: ¿No te consideras una guarra (1) como quien?

Pretendienta Laia tronista Alex.: Como todas

Pretendienta Carla tronista Alex.: (...) dejas entrar a todas las guarras $<$ de de de $>$ todos lados y encima $\uparrow$ ipretendes que yo me quede $\uparrow$ ?

En las dos primeras referencias anteriores, observamos como se recoge la idea de profundidad en las relaciones, donde se conoce lentamente o realmente al otro. En la tercera referencia, con la crítica todo el mundo busca solo que follar y todo eso al igual que destacamos anteriormente, hace referencia a la incursión del repertorio líquido en la sociedad actual, por tanto, reconoce una esencia de comportamiento líquido, y muestra rechazo a aquellas personas que actúen conforme a dicha esencia. En este caso concreto, rechaza la superficialidad líquida de no estrechar vínculos. Como hemos ido observando, se genera una clasificación dual, que permite calificar como positivo aquello que se incluye en el repertorio romántico, y descalificar lo que se inserta en el líquido. Asimismo, la inclusión de la parte (en este caso las personas 
afines al repertorio líquido) en el todo, todo el mundo, otorga mayor validez a su intervención.

Vamos a detenernos y a analizar en profundidad, desde la perspectiva de género la penúltima referencia. En ella, la pretendienta establece un enlace simbólico entre el símbolo (guarra) y lo simbolizado (mujer que expresa características líquidas). El uso metonímico del término guarra, sustituye a mujer líquida. Este enlace suscita amor y veneración a las personas que realizan prácticas románticas (recordemos la intervención muy bien, encima, encima romántica) y odio y desprecio a las prácticas líquidas (recordemos las anteriores referencias analizadas donde esto se hace presente). Al ser una máxima el repertorio romántico, no es de extrañar que este enlace simbólico no provocase más que risas en los allí presentes. Se presenta a ella misma como modelo, el cual indica la conducta a seguir, modelo de los valores románticos, frente al antimodelo, guarras. Los ejemplos de mujeres antimodelo que aparecen en el programa, son cuestionadas o expulsadas inmediatamente. Todo ello contribuye a seguir reproduciendo el discurso del amor romántico como ideal y el del amor líquido como deplorable. La crítica a estas formas líquidas reconoce una esencia actual de comportamiento líquido, ante la cual quiere desmarcarse, mostrarse con valor de unicidad, no me considero como, como una guarra de las tantas que hay. Cuando hace referencia a como todas, expresa una sinécdoque y un argumento cuasilógico de frecuencia, designar la parte por el todo, y reforzar de nuevo su valor como modelo y de unicidad. Toda su intervención, es un proceso de disociación, de separar elementos considerados dentro de un todo, el todo líquido y sus elementos guarras, y el todo romántico y sus elementos las mujeres románticas, como ella se presenta. Asimismo, observamos cómo las mismas mujeres presentan rechazo a las otras mujeres que abogan por mantener relaciones superficiales y libertarias. En ningún momento del material analizado se encuentra una palabra o expresión que estigmatice a los hombres por llevar a cabo prácticas líquidas, en cambio el apelativo guarra aparece varias veces a lo largo del material, para referirse a las mujeres que mantienen relaciones líquidas (superficiales y libertarias) con otros. Esto que aquí analizamos trae a colación las palabras de Carol Smart (2000), donde sostiene que la mujer representa el dualismo, en el sentido de que en el discurso jurídico, la prostituta es construida como la mala mujer, o en este caso, guarra es construida, es símbolo de mala mujer, pero al mismo tiempo se erige en el epítome de la Mujer en contraposición al Hombre, porque es lo que cualquier mujer podría ser, y porque representan una tortuosidad y un libertinaje surgidos de su forma corporal (que se supone naturalmente dada), mientras que el hombre permanece inofensivo.

A pesar del continuo rechazo a las formas superficiales y libertarias de la liquidez, se nos presentan ejemplos donde se reproducen características propias de la superficialidad líquida, sin que se muestre rechazo por ello:

(...) Me gustan las chicas que tengan e:::, aire así pijillo, (...) mm::: soy muy superficial (1) demasiado creo. (Tronista Carles)

Presentador.: Muy bien Roberto (2) primer impacto Laura

Tronista Laura.: ((ríe)) es que no quiero ser maleducada

(...)

Presentador.: Decide, ¿quieres que esté o despedimos?

Tronista Laura.: Siento mucho decirlo, pero no 
Presentador:: ¿Te quedas? ¿Carles, te gusta?

Tronista Carles.: Mm:::(4) no se ((hace gestos con las manos y le echa una mirada de arriba a abajo))

Presentador.: (...) Para presentar a la siguiente y para continuar el programa debes decir si se queda o se tiene que ir

Tronista Carles.: ¿No puede ser cuando pase alguna más?

(...)

Presentador:: ¿entonces la dejas o no la dejas?

Tronista Carles.: Déjala, pero:.: quiero que pase alguna más

Presentador.: La dejo, la dejo (...)

Tronista Carles.: A primera impresión es como una mini Barbie

Presentador.: $\uparrow U n a$ mini Barbie $\uparrow$, una mini Barbie

(...)

Presentador:: Una mini Barbie

Desde un análisis de género, en las dos últimas referencias, se presenta a la mujer como un ente sin agencia. En la penúltima referencia, se somete a las voluntades de su comprador, el cual decide sobre su devenir. En el último ejemplo se presenta a la mujer como una muñeca, objeto frágil, manipulable, manejable, pasivo, subordinado a las manos de quien la posee. Ya no solo referirse a ella de esta manera, sino que se amplifica este sentido de inferioridad con la expresión mini que es repetida las mismas cuatro veces que Barbie. Prestamos especial atención a que el presentador lo repite tres veces, siendo su palabra argumento de autoridad. Con el uso del conduplicatio, al repetir más de una vez la misma expresión, no solo duplica el efecto de presencia, sino que el último enunciado está cargado de valor. A pesar de ser Barbie una muñeca de unos 20 centímetros, tanto tronista como presentador con sus continuas repeticiones le dan una subordinación aún menor que a la muñeca. Bourdieu (2001) sostiene que las construcciones sobre masculinidad y feminidad se escriben sobre el cuerpo, la mujer es objetivizada sexualmente, se la separa del resto de su personalidad, y de esta forma, se la aísla del todo y queda reducida a instrumentos que logran representar al sujeto dominado (Bartky, 1990).

Además, como ya comentamos en el eje de Compromiso/Libertad, las dos pretendientas que acudieron a conocer a tronista Carles en el primer programa, no aparecieron en el segundo. Y de las tres nuevas que entraron al segundo programa, solo una apareció en el tercero. Esta situación denota falta de profundidad, siendo la profundidad, una característica que destaca en el repertorio romántico, necesaria para poder conocer realmente al otro.

El próximo eje está muy relacionado con el que acabamos de presentar, a pesar de que las referencias del mismo podrían utilizarse como claros ejemplos de este apartado, no ocurre lo mismo al contrario, y es por esto que hemos querido presentarlo a parte pues aunque muy similares, constituyen categorías diferentes.

\subsection{Realidad/Virtualidad}

Con estas categorías, queremos recoger la influencia que tienen las redes a la hora de relacionarnos, a pesar de esto, sigue la idea romántica de que la forma correcta 
o verdadera de encontrar el amor, es en persona. A pesar de como señala Bauman (2003), respecto a las posibilidades que nos aportan las relaciones virtuales, de fácil acceso y salida, el discurso de los jóvenes de este programa muestra que este acceso y salida, debe hacerse correcta o verdaderamente en persona, como en las relaciones convencionales.

A diferencia de en los apartados anteriores, en aras de facilitar la comprensión del lector, presentaremos en primer lugar las referencias a la categoría asociada al amor líquido (virtualidad):

Pretendienta Silvia tronista Carles.: No sé, o sea, me gusta su físico, su personalidad, de lo poco que le he visto en $A s k$, o sea (...)

Pretendiente Mateu tronista Paula.: La estaba mirando en Facebook, pues no se, me gustó

Pretendienta Carol tronista Alex: Lo vi en Facebook y me pareció muy guapo y pues aquí estoy

Las redes sociales, son en sí misma una herramienta actual para relacionarse, a pesar de esto, se usan como primera toma de contacto, como un acercamiento a la persona que nos atrae, sin profundizar en ella. Posteriormente, se acude al programa para en persona poder tener una relación de verdadero amor:

Laura viene (...) a conocer de verdad el amor (tronista Laura)

(...) ¿qué esperas del programa? ¿conocer, encontrar el amor? (presentador)

Pretendiente Oriol tronista Paula.: (...) Porque en la calle no he tenido mucha oportunidad y quiero conocerla de verdad

Los lugares de la existencia confirman la superioridad de lo real, lo que existe, lo que es actual. El amor se presenta como ente externo, real, que existe, que espera ser conocido.

Podemos observar en los apartados anteriores que esta libertad/superficialidad y falta de compromiso/profundidad en las relaciones, muestra un fácil acceso y salida de las relaciones, dando la sensación de estarnos refiriendo a relaciones virtuales y no convencionales en persona. Entendemos que esto es debido al proceso de transición y por tanto de convivencia del repertorio líquido y romántico, y como observamos en este caso, fusionándose características de cada repertorio y produciéndose relaciones convencionales cara a cara, pero con fácil acceso y salida, como ocurriría en las relaciones virtuales.

\section{Conclusiones}

A pesar de tratarse de un programa diseñado bajo el repertorio del amor romántico, las formas románticas que se esperan o manifiestan, entran en continuo conflicto con las manifestaciones líquidas. Somos conscientes que el repertorio del amor romántico orienta sobre las formas adecuadas de sentir, produciendo y sustentando prácticas discriminatorias que sitúan lo femenino sexualizado y subordinado a lo masculino, fomentando y permitiendo esta desigualdad entre hombres y mujeres, y 
posibilitando la aparición y mantenimiento de la violencia de género en sus distintas formas. Aunque en menor medida, el repertorio del amor líquido no parece producir algo muy diferente, la mujer no solo soporta ser objetivizada sexualmente cual ente de consumo, sino que también se la culpa, responsabiliza y estigmatiza por ello - no olvidemos las referencias al término guarra que aparecen a lo largo del análisis-, y aquellas que mantienen relaciones hedónicas, superficiales y libertarias, y por tanto, prácticas líquidas, son rechazadas socialmente. El repertorio líquido se presenta como una amenaza hacia la hegemonía del repertorio romántico, y por ende, la liquidez es rechazada socialmente, así como a las personas que muestran características asociadas a este repertorio. Las mujeres son más críticas con este repertorio y con las mujeres que muestran características del mismo, los hombres, aunque muestran rechazo social al repertorio líquido, se muestran más cómodos a la hora de comportarse de forma líquida. De este modo, al igual que el amor romántico sitúa al hombre en una situación de poder frente a la subordinación de las mujeres, el repertorio líquido conduce a algo similar. Estos resultados se corresponden con las reflexiones presentadas en la introducción, sobre la necesidad de que las mujeres lleguen a ser parte activa en la construcción de un modelo relacional y unas relaciones sexo afectivas igualitarias, y se rechace su subordinación en las mismas; antes que centrarnos en si estrechar más o menos unos lazos relacionales, va a significar un mayor o menor trato diferencial entre mujeres y hombres.

Los discursos que emergen del material analizado no pueden desprenderse de un único repertorio. Las prácticas románticas nos orientan a comprometernos en el mantenimiento de relaciones profundas, a dejarnos llevar por la emoción, a sacrificarnos por el otro, considerando estas, características de real o verdadero amor. Por el contrario, las prácticas líquidas, abogan por relaciones libertarias, superficiales y hedónicas, abordadas de forma racional, siendo prácticas comunes en las relaciones virtuales. Asimismo, como muestran los resultados, no solo conviven estas prácticas, sino que en cierto modo se complementan, observándose prácticas emocionales para relaciones superficiales; prácticas de sacrificio para relaciones libertarias; prácticas físicas, y por tanto consideradas reales, mantenidas bajo prácticas superficiales, libertarias y hedónicas.

Los resultados de este trabajo nos plantean considerar la emergencia de un nuevo fenómeno al cual hemos denominado Consumo Romántico en el cual se complementan, conviven y fusionan características de los repertorios romántico y líquido. Este fenómeno recoge la convivencia de relaciones emocionales, comprometidas, sacrificadas, profundas y reales, con relaciones racionales, libres, hedónicas, superficiales y virtuales. Asimismo, nos muestra cómo se complementan estas categorías entre ellas, produciendo relaciones emocionales y racionales, profundas y hedónicas, comprometidas y hedónicas, emocionales y superficiales, comprometidas y racionales, reales y superficiales. Esta complementariedad entre características principalmente separadas por su adscripción a un repertorio concreto, se fusionan para generar actos emocionales, irracionales que se llevan bien con las consideraciones técnicas; relaciones convencionales cara a cara de fácil acceso y salida, como ocurre en las relaciones virtuales; relaciones profundas y comprometidas donde se evita el sacrificio que requiere la exclusividad, sobre todo a medio-largo plazo, y se analiza racionalmente esta falta de sacrificio o de compromiso, traduciéndose en la valoración positiva de las relaciones exclusivas y comprometidas, siempre y cuando sean hedónicas, y por tanto, podríamos afirmar que este nuevo fenómeno nos mues- 
tra cómo las relaciones románticas monógamas no tienen mucha vida más allá de la fase de enamoramiento, considerada esta la fase más hedónica del amor.

\section{Referencias}

Bartky, Sandra Lee (1990). Femininity and domination: Studies in the phenomenology of oppression. Psychology Press.doi:10.4324/9780203825259

Bauman, Zygmunt (2003). Amor líquido: acerca de la fragilidad de los vínculos humanos. Fondo de Cultura Económica.

Bauman, Zygmunt (2007). Vida de consumo. Buenos Aires: Fondo de la Cultura Económica.

Bourdieu, Pierre (1998/2001). Masculine domination. Stanford University Press. https://doi. org/10.2307/40184116

Coral-Díaz, Ana Milena (2010). El cuerpo femenino sexualizado: entre las construcciones de género y la Ley de Justicia y Paz. International Law, (17), 381-409.

Cubells, Jenny y Calsamiglia, Andrea (2016). El repertorio del amor romántico y las condiciones de posibilidad para la violencia machista. Universitas Psychologica 14(5), 16811694. doi:10.11144/Javeriana.upsy14-5.rarc

De Beauvoir, Simone (1949/1969). El segundo sexo. Buenos Aires: Siglo Veinte.

De Miguel, Ana (2015). Neoliberalismo Sexual: El mito de la libre elección. Madrid: Feminismos

España (2006). Real decreto legislativo 1/1996, de 12 de abril, por el que se aprueba el Texto refundido de la Ley de propiedad intelectual, regularizando, aclarando y armonizando las disposiciones legales vigentes sobre la materia. Boletín Oficial del Estado. Madrid, 22 de abril de 1996, núm. 97. https://www.boe.es/buscar/act.php?id=BOE-A-1996-8930

Estalella, Adolfo y Ardévol, Elisenda (2007). Ética de campo: hacia una ética situada para la investigación etnográfica de internet. In Forum Qualitative Social Research (Vol. 8, No. 3 , p. 25).

Esteban, Mari Luz y Távora, Ana (2008). El amor romántico y la subordinación social de las mujeres: revisiones y propuestas. En Anuario de Psicología 39(1) 59-73.

[FilmNight Barcelona]. (2015, abril 28). Chicos Y Chicas - EPISODIO 1 (Parte 1/2) [Archivo de video]. Recuperado de https://www.youtube.com/watch?v=tBv2c-KOTz4.

[FilmNight Barcelona]. (2015, abril 28). Chicos y Chicas - EPISODIO 1 (parte 2/2) [Archivo de video]. Recuperado de https://www.youtube.com/watch?v=DYgN6Wgasrc

[FilmNight Barcelona]. (2015, mayo 5). Chicos Y Chicas - EPISODIO 2 (Parte 1/2) [Archivo de video]. Recuperado de https://www.youtube.com/watch?v=0jIWy9BIp4c

[FilmNight Barcelona]. (2015, mayo 7). Chicos Y Chicas - EPISODIO 2 (Parte 2/2) [Archivo de video]. Recuperado de https://www.youtube.com/watch?v=ewujqcX2A3E

[FilmNight Barcelona]. (2015, mayo 14). Chicos Y Chicas - EPISODIO 3 (Parte 1/1) [Archivo de video]. Recuperado de https://www.youtube.com/watch?v=P1I0qPiRrJ0

[FilmNight Barcelona]. (2015, mayo 25). Chicos Y Chicas - EPISODIO 4 (mini) [Archivo de video]. Recuperado de https://www.youtube.com/watch?v=NvVLH-ce_k8

[FilmNight Barcelona]. (2015, junio 3). Chicos Y Chicas - EPISODIO 5 [Archivo de video]. Recuperado de https://www.youtube.com/watch?v=H_2uhYULg6Y

[FilmNight Barcelona]. (2015, junio 10). CHICOS Y CHICAS - [ALEX \& MARTA] [Archivo de video]. Recuperado de https://www.youtube.com/watch? $=$ GysgHMD5Tqc 
[FilmNight Barcelona]. (2015, junio 22). Chicos Y Chicas - EPISODIO 6[Archivo de video]. Recuperado de https://www.youtube.com/watch?v=MrFtskyL-GQ_

Follari, Javier Ernesto B. (2015). El código de transcripción de Gail Jefferson: adaptación para las ciencias sociales. Quaderns de psicologia. International journal of psychology, 17(1), 39-62

Foucault, Michel (1992). El orden del discurso [1970]. Buenos Aires: Tusquets EditoresFriedan, B. 1963/2009. La mística de la feminidad. Ediciones Cátedra.

Gergen, Kenneth J. (2006). El yo saturado: dilemas de identidad en el mundo contemporáneo. Paidós Ibérica. doi:10.1515/9781400848393-062

Gergen, Kenneth J. (2007). Constructivismo social: aportes para el debate y la práctica. Universidad de los Andes, Facultad de Ciencias Sociales.

Gergen, Kenneth J. \& Gergen, Mary M. (2011). Reflexiones sobre la construcción social. Paidós.

Gil, Eva Patricia y Lloret, Imma (2007). La violencia de género. Barcelona: UOC.

Haraway, Donna. (1988) Situated Knowledges: The Science Question in Feminism as a Site of Discourse on the Privilege of Partial Perspective. Feminist Studies 14, no.3 p.p: 575 99. doi:10.2307/3178066

Harré, Rom (1986). The social construction of emotions. Oxford: Blackwell.

Harré, Rom y Parrott, Gerrod W. (1996). The emotions: Social, cultural and biological dimensions. Londres: Sage.

Hooks, Bell (2000). All about love: New visions. William Morrow.

Illouz, Eva (2009). El consumo de la utopía romántica: El amor y las contradicciones culturales del capitalismo. Katz Editores.

Iñiguez, Lupicinio (2008). El debate sobre metodología cualitativa versus cuantitativa. $M a-$ terial preparado para la Maestría en Ciencias Sociales. Universidad de Guadalajara: Guadalajara.

Iñiguez, Lupicinio y Antaki, Charles (1994). El análisis del discurso en psicología social. Boletín de psicología, 44, 57-75.

Jónnasdóttir, Anna (1996). El poder del amor: ¿le importa el sexo a la democracia?. Madrid: Cátedra.

Krippendorff, Klaus (1990). Metodología de análisis de contenido: teoría y práctica. Ediciones Paidós Ibérica.

Kollontai, Alejandra (1976). Marxismo y revolución sexual. Madrid

Lagarde, Marcela (2001) Claves feministas para la negociación en el amor. Managua: Puntos de Encuentro.

Lizcano, Emmánuel (1999). La metáfora como analizador social. Revista de Metodología de Ciencias Sociales, (2), 29-60. doi:10.5944/empiria.2.1999.709

Lizcano, Emmánuel (2006). Metáforas que nos piensan: sobre ciencia, democracia y otras poderosas ficciones. Madrid: Ediciones Bajo Cero / Traficantes de sueños. doi: 10.5944/ empiria.11.2006.1117

Medina Doménech, Rosa M. (2012). Ciencia y sabiduría del amor: una historia cultural del franquismo. Madrid-Frankfurt: Iberoamericana-Vervuert. https://doi.org/10.6018/daimon/196671

Olsen, Frances (2000). El sexo del derecho. En Ruiz, Alicia y Amorós, Celia (Eds.). Identidad femenina y discurso jurídico. Buenos Aires: Biblos

Perelman, Chaïm y Olbrechts-Tyteca, Lucie (1976/1989). Tratado de la argumentación : la nueva retórica. Madrid: Gredos. Potter, J. \& Wetherell, M. 1987. Discourse and social psychology: Beyond attitudes and behaviour. London: Sage. 
Pomerantz, Anita (1986). Extreme case formulations: A way of legitimizing claims. Human Studies, 9, 219-229. doi:10.1007/bf00148128

Pujal, Margot (2007). El Feminisme. Barcelona: UOC.

Sánchez-Sicilia, Alejandro y Cubells, Jenny (2017). Discurso amoroso adolescente: Análisis del repertorio del amor romántico en el programa chicos y chicas. Athenea digital: En prensa.

Smart, Carol (2000). La teoría feminista y el discurso jurídico. Biblos 31-71.

Vázquez Sixto, Felix (1996) El análisis de contenido temático. Documento de trabajo Objetivos y medios en la investigación psicosocial 47-70. Universitat Autònoma de Barcelona. Barcelona

Wetherell, Margaret y Potter, Jonathan (1996). El análisis del discurso y la identificación de los repertorios interpretativos. En Ángel J. Gordo y José Luis Linaza (eds.). Psicologías, discurso y poder (PDP) (pp.63-78). Madrid: Visor

Wolgar, Steve (1988). Science: the Very Idea. Chichester, Ellis Horwood; Londres, Tavistock. 